\title{
Combined effect of palygorskite and melamine polyphosphate on flame retardancy properties of polyamide 11 nanocomposites
}

\author{
Baya Benobeidallah $^{\mathrm{a}, \mathrm{b}, *}$, Aida Benhamida ${ }^{\mathrm{c}}$, Mustapha Kaci ${ }^{\mathrm{a}}$, José-Marie Lopez-Cuesta ${ }^{\mathrm{b}}$ \\ ${ }^{\mathrm{a}}$ Laboratoire des Matériaux Polymères Avancés (LMPA), Université de Bejaia 06000, Algeria \\ ${ }^{\mathrm{b}}$ Centre des Matériaux des Mines d'Alès (C2MA), IMT-Mines d'Alès, 6 avenue de Clavières 30319, Alès, Cedex, France \\ ${ }^{\mathrm{c}}$ Laboratoire des Silicates, des Polymères et des Nanocomposites (LSPN), Université de Guelma 24000, Algeria
}

\begin{abstract}
A B S T R A C T
Pyrolysis and fire behaviour of palygorskite (Pal) fibrous clay (5 wt \%) reinforced polyamide 11 nanocomposites prepared by melt compounding were investigated in combination with melamine polyphosphate (MPP). Palygorskite was modified by 3-amino-propyl-triethoxysilane (APTES). Thermal stability and combustion behaviour of the materials were studied by thermogravimetric analysis (TGA), pyrolysis combustion flow calorimeter (PCFC) and cone calorimeter. TGA data showed that MPP reduced the thermal stability of PA11 nanocomposites, whereas the addition of both unmodified and modified palygorskite improved this property. Further, the cone calorimeter and PCFC results indicated a significant decrease in the peak of heat release rate (pHRR) and the total heat release (THR) of PA11 nanocomposites. This also means that the PA11 combustion was strongly reduced in the presence of combined Pal with MPP, being however more pronounced for the modified palygorskite. The improved fire behaviour could be attributed to a synergistic effect between Pal and MPP.
\end{abstract}

Keywords:

Polyamide 11

Palygorskite

Nanocomposite

Melamine polyphosphate

Flame retardancy

\section{Introduction}

Polyamide 11 (PA11) is an important commercial polymer obtained from renewable resources (castor oil). It is an attractive polyamide used as an engineering polymer in various industrial sectors from automotive to offshore oilfield applications (Kaci et al., 2019). This is due to its excellent performance related to low moisture sorption, good chemical resistance and easy processability (Zhang et al., 2004, 2012). However, the use of PA11 is limited by its poor heat resistance and fire performances. Therefore, to extend the PA11 applications in industrial sectors such as electric, electronics, construction and transportation, which require specific fire safety, its fire properties need to be improved.

Many strategies have been reported in the literature to reduce the flammability of polyamide 11 (Koo et al., 2008; Lao et al., 2009; Lao et al., 2010b; Hao et al., 2015; Jin et al., 2016; Negrell et al., 2016; Macheca et al., 2017; Sahnoune et al., 2018). Accordingly, halogen-free flame retardants including metal hydroxides, phosphorous and nitrogenous compounds are usually required to achieve good fire performances. These flame retardants (FR) can act through fuel dilution in the gaseous phase and/or formation of a charred barrier in the condensed phase (Braun et al., 2007; Rakotomalala et al., 2010). This charred structure can act as an insulator to the underlying material against the diffusion of heat, oxygen and gaseous decomposition products, thus decreasing the flammability of the materials. High amounts of flame-retardant additives ( $>30 \mathrm{wt} \%$ ) are often necessary to achieve required fire performances. Nevertheless, the use of FRs can lead to a reduction of the mechanical properties of the materials (Levchik et al., 1992; Lao et al., 2010b).

Recently, the incorporation of nanofillers such as modified montmorillonite, halloysite, silica and carbon nanofibers to PA11 matrix, offers a promising method to improve its fire properties (Koo et al., 2008; Lao et al., 2010b; Lao et al., 2011; Hao et al., 2015; Macheca et al., 2017; Sahnoune et al., 2018). In this regard, Sahnoune et al. (Sahnoune et al., 2018) reported that the addition of $20 \mathrm{wt} \%$ of halloysite modified with methyl phosphonic acid led to a better reduction in the peak of heat release rate (pHRR) determined by cone calorimeter test. However, the nanoparticles cannot be used alone as flame retardant (FR) to meet requirements of fire reaction standards (Lao et al., 2011; Yang and Nelson, 2011). Indeed, several studies reported the combination of different types of nanofillers and FR additives, including hydrated minerals (Lao et al., 2010a; Lao et al., 2011), phosphorous compounds (Jin et al., 2017; Sahnoune et al., 2018) or intumescent FR systems (Hao et al., 2015) in order to highlight the existence of synergistic effects on fire properties.

Palygorskite (Pal) is a kind of 2:1 hydrated magnesium aluminium silicate with fibrous morphology. Pal has a structure composed of

\footnotetext{
* Corresponding author at: Laboratoire des Matériaux Polymères Avancés (LMPA), Université de Bejaia 06000, Algeria.

E-mail address: benobaya@gmail.com (B. Benobeidallah).
} 
ribbons, which are parallel to the fiber axis (Qi et al., 2013). Pal exhibits excellent chemical and thermal stability, large specific surface area, high aspect ratio and high density of silanol groups (Zhang et al., 2014; Belaroui et al., 2018). Due to these interesting properties, Pal is widely used as reinforcing for polymeric materials (Pan et al., 2006; Yuan et al., 2007; Chen et al., 2011)' as well as flame retardant on various polymer matrices such as polystyrene (Yang and Nelson, 2011), polypropylene (Su et al., 2011), rubber (Rybiński et al., 2013), polyamide 6 (Hou et al., 2018). The literature data clearly reported the effectiveness of the combination approach in the preparation of flameretardant composites.

In this context, Su et al. (Su et al., 2011) showed that the incorporation of only $2 \mathrm{wt} \%$ of Pal with $24 \mathrm{wt} \%$ of intumescent flame retardant system (IFR) in polypropylene led to much higher fire performances than PP/IFR composites. The authors argued that the active sites of palygorskite could catalyse the formation of the protective char, which significantly e nhanced the fi re pr operties of PP /IFR systems. Yang et al. (Yang and Nelson, 2011) reported that adding $3 \mathrm{wt} \%$ of $\mathrm{Pal}$ induced a reduction in pHRR of polystyrene by $13 \%$, while the combination of Pal with flame retardant containing polysiloxane and borate significantly reduced the pHRR by $44 \%$.

Although, the literature data is abundant on the fire properties of engineering polymers filled with Pal, however, no paper is available yet on PA11/Pal nanocomposite systems. Therefore, the main objective of this work was to investigate the potential use of Algerian palygorskite as the flame retardant in PA11. The nanocomposite samples of PA11 filled with palygorskite (Pal) and silane treated palygorskite (sPal) with and without melamine polyphosphate flame $r$ etardant ( M PP)were prepared by melt compounding in a twin-screw extruder. Thermal stability of the materials was investigated by TGA, while cone calorimeter test and Pyrolysis-combustion flow calorimetry (PCFC) were used to examine the fire properties of the samples. The Energy Dispersive Xray spectroscopy (EDS) was used to determine the elemental composition of the residue after burning and the crystalline structure was analyzed by wide angle X-ray diffraction (WAXD).

\section{Experimental}

\subsection{Materials}

Commercial polyamide 11 (PA11, RILSAN ${ }^{\circledR}$ LMNO) was supplied by ARKEMA (France). The polymer has a semi-crystalline structure and its main physical characteristics are: a melting point of $186{ }^{\circ} \mathrm{C}$ and a density of $1.02 \mathrm{~g} / \mathrm{cm}^{3}$. Melamine polyphosphate (MPP, Melapur ${ }^{\circledast 2} 200$ ) supplied by BASF (Germany) was used as the flame retardant. It has a density of $1.85 \mathrm{~g} / \mathrm{cm}^{3}$ and a thermal decomposition temperature above $350{ }^{\circ} \mathrm{C}$. The fibrous clay used is Algerian Palygorskite extracted from Ghoufi's deposit located in the Eastern region of Algeria. It was purified by acetic acid at a low concentration $(<3 \mathrm{M})$ to remove the impurities and to generate a high number of silanol groups on the surface (FriniSrasra and Srasra, 2010). Then, the purified palygorskite was subjected to a chemical treatment, consisting of grafting organic APTES molecules on the surface of fibrous clay in order to improve the interactions between the organic and inorganic compounds. The details on the grafting process are provided in the next section.

\subsection{Functionalization process}

$50 \mathrm{~g}$ of palygorskite powder and $5 \mathrm{ml}$ of silanes coupling agent, i.e., 3-aminopropyltriethoxy silane (APTES), were dispersed in $500 \mathrm{ml}$ of ethanol/water (98/2) solution. The mixture was refluxed for $24 \mathrm{~h}$ at $80{ }^{\circ} \mathrm{C}$ under magnetic stirring. The product was separated by centrifugation and washed with ethanol to remove the excess of silanes. The silanized palygorskite (sPal) was dried in a vacuum at $40{ }^{\circ} \mathrm{C}$ for 24 h.
Table 1

Codes and compositions in wt $\%$ of the different formulations used.

\begin{tabular}{lllll}
\hline Samples & PA11 (wt\%) & Pal (wt\%) & MPP (wt\%) & sPal (wt\%) \\
\hline PA11 & 100 & 0 & 0 & 0 \\
PA11-Pal & 95 & 5 & 0 & 0 \\
PA11-sPal & 95 & 0 & 0 & 5 \\
PA11-MPP & 75 & 0 & 25 & 0 \\
PA11-Pal-MPP & 75 & 5 & 20 & 0 \\
PA11-sPal-MPP & 75 & 0 & 20 & 5 \\
\hline
\end{tabular}

\subsection{Melt compounding}

Prior to compounding, neat PA11 and Pal were dried under vacuum at $80{ }^{\circ} \mathrm{C}$ for $24 \mathrm{~h}$. The polymer/filler nanocomposites were prepared by melt compounding under vacuum in a twin-screw extruder (Clextral BC21) with a screw diameter (D) of $25 \mathrm{~mm}$ and length to diameter ratio (L/D) of 48. The screw speed was fixed at $250 \mathrm{rpm}$ and the temperature was ranged between 190 and $230{ }^{\circ} \mathrm{C}$. The sample codes and weight compositions are provided in Table 1 . Square specimens with dimensions of $100 \times 100 \times 4 \mathrm{~mm}$ for cone calorimeter test were prepared by injection moulding using a 50-ton Krauss-Maffei equipment (Munich, Germany). The temperature profile was set from 220 to $230{ }^{\circ} \mathrm{C}$ and the mould temperature was $40{ }^{\circ} \mathrm{C}$. The injection pressure and the rotation speed were kept at 135 bar and $100 \mathrm{rpm}$, respectively.

\subsection{Characterization techniques}

The thermal stability of both palygorskite and PA11 nanocomposite samples was determined by TGA, using a Setsys TGA, Setaram instruments. Palygorskite was heated from 25 to $900{ }^{\circ} \mathrm{C}$ at a heating rate of $10{ }^{\circ} \mathrm{C} / \mathrm{min}$, while PA11 nanocomposite samples were heated up to $700{ }^{\circ} \mathrm{C}$. The TGA tests were performed under nitrogen and air atmospheres. The average weight of samples was almost $10 \mathrm{mg}$. PCFC analysis was performed in nitrogen atmosphere (method A: anaerobic conditions) and in a mixture of nitrogen and oxygen (method B: aerobic conditions) according to ASTM D7309 using Fire Testing Technology (FTT) apparatus at a heating rate of $1{ }^{\circ} \mathrm{C} / \mathrm{s}$. 2-3 $\mathrm{mg}$ of samples were heated to $750{ }^{\circ} \mathrm{C}$ corresponding to the maximum pyrolizer temperature and the combustor temperature was $900{ }^{\circ} \mathrm{C}$. The flow in the combustor was a mixture of oxygen/nitrogen (20/80) at $100 \mathrm{~cm}^{3} / \mathrm{min}$. The cone calorimeter experiments were performed on square samples having the dimensions of $100 \times 100 \times 4 \mathrm{~mm}$, using FTT apparatus. Irradiance source was set at 35 and $50 \mathrm{~kW} / \mathrm{m}^{2}$ for all samples. Time to ignition (TTI), peak of heat release rate (pHRR) and total heat release (THR) were determined. The surface modification of palygorskite before and after grafting the APTES molecules on the fibrous surface and the chemical composition of the residue after the cone calorimeter test was characterized by Fourier Transform Infrared (FT-IR) spectroscopy in ATR mode. The FT-IR spectra were recorded using a Bruker spectrometer. The wavenumber was ranged from 400 to $4000 \mathrm{~cm}^{-1}$ with a resolution of $2 \mathrm{~cm}^{-1}$. The WAXD patterns of the palygorskite and the cone calorimeter residue were recorded using a Bruker AXS D8 Advance diffractometer (Bruker, USA) equipped with $\mathrm{CuK} \alpha$ radiation (1.5418 $\mathrm{A}^{\circ}$ ). The diffraction angle ranged from $2 \theta=5^{\circ}$ to $60^{\circ}$ with a step size of $0.007^{\circ}$. The elemental composition of the residue from the cone calorimeter test was evaluated by Energy Dispersive X-ray spectroscopy (EDS) using Oxford INCA Energy system endowed with a probe of $80 \mathrm{~mm}^{2}$ and a detector of $133 \mathrm{ev}$.

\section{Results and discussion}

\subsection{Characterization of the Pal surface after APTES treatment}

Fig. 1 shows the FT-IR spectra of $\mathrm{Pal}$ and $\mathrm{sPal}$ recorded in $4000-800 \mathrm{~cm}^{-1}$ and $4000-2700 \mathrm{~cm}^{-1}$ regions. In the broader FT-IR 


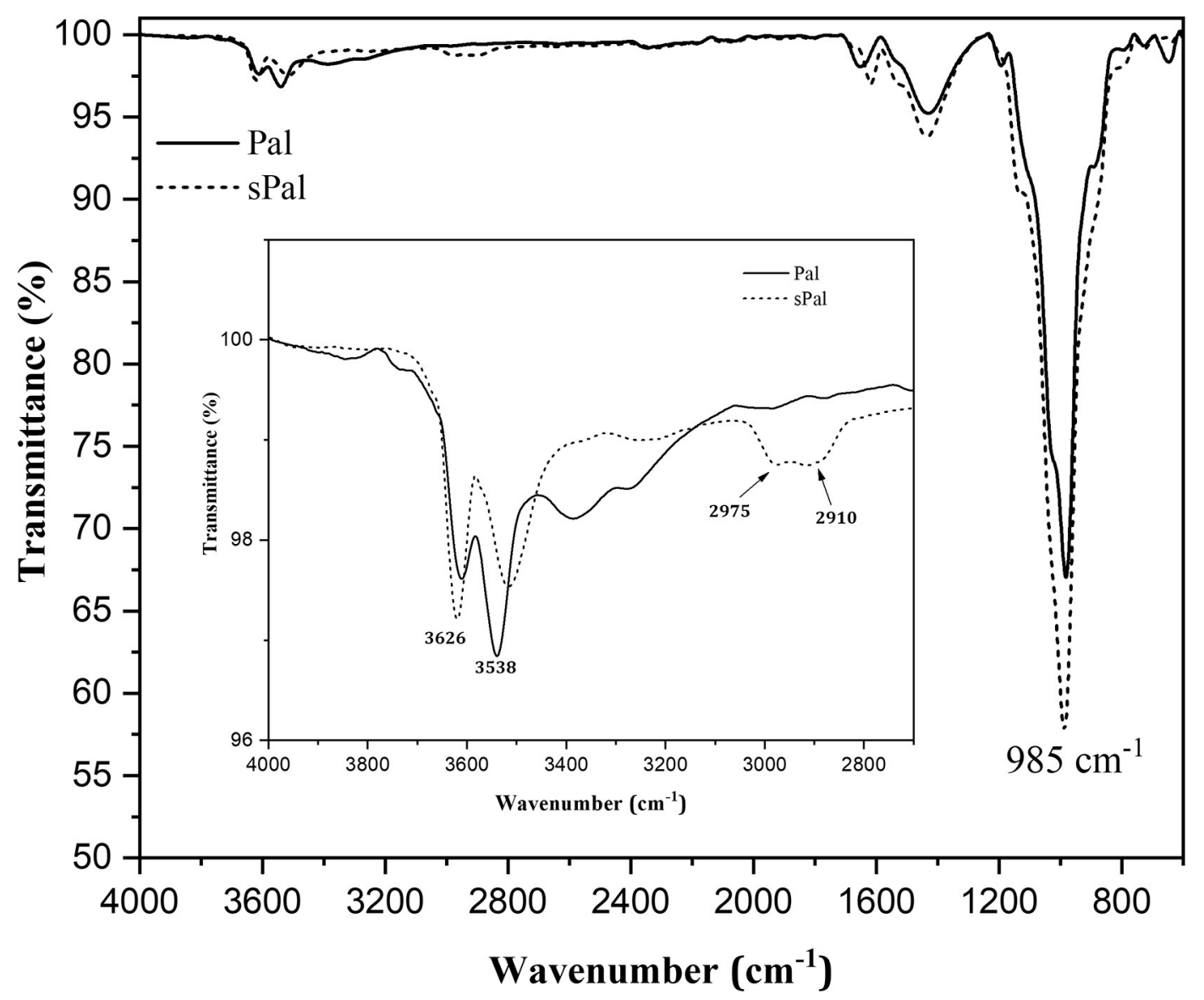

Fig. 1. FT-IR spectra of palygorskite before and after organo-silane treatment.

spectra, the main absorption band is observed at almost $985 \mathrm{~cm}^{-1}$. The latter is assigned to the $\mathrm{Si}-\mathrm{O}-\mathrm{Si}$ vibration in the palygorskite structure (Moreira et al., 2017). The band intensity at $985 \mathrm{~cm}^{-1}$ increases after APTES treatment of Pal. Also, it appears clearly in the $4000-2700 \mathrm{~cm}^{-1}$ region, the occurrence of significant changes in the chemical structure of Pal after treatment, which are localized at around 2910 and $2975 \mathrm{~cm}^{-1}$. The corresponding absorption bands are assigned to the stretching vibration of $-\mathrm{CH}_{2}$ groups of APTES (Wang et al., 2014), which indicate the presence of APTES molecules on the Pal surface. The bands observed at $3626,3538,3400$ and $3250 \mathrm{~cm}^{-1}$ are attributed to the hydroxy groups of $\mathrm{Si}-\mathrm{OH}$, crystalline water, zeolitic water and adsorption water, respectively (Zhang et al., 2014). The band intensity decreases after APTES treatment indicating the substitution of the hydroxyl groups of Pal by the APTES molecules.

TGA thermograms of $\mathrm{Pal}$ and sPal shown in Fig. 2 display a continuous mass loss for both clay samples. The mass loss recorded up to $480{ }^{\circ} \mathrm{C}$ corresponds to the elimination of zeolitic and coordinated water in the fibrous structure. Whereas, the mass loss at $737^{\circ} \mathrm{C}$ is due to the de-hydroxylation of the palygorskite (Chen et al., 2012). In addition, the sPal sample shows an additional endothermic peak at $416^{\circ} \mathrm{C}$, which is assigned to the thermal decomposition of grafted APTES molecules (Zhu et al., 2016; Moreira et al., 2017). TGA data indicate that the mass loss of Pal is about $24 \%$ at $900{ }^{\circ} \mathrm{C}$, which slightly increases to $25.4 \%$ for sPal. This small increase in the mass loss of sPal compared to Pal is due probably to grafting of APTES molecules on palygorskite surface.

The FT-IR results are consistent with the TGA data. Indeed, the reduction of the band intensity of hydroxyl groups of Pal in FT-IR spectra after APTES treatment associated with the decrease of the first mass loss of Pal in the presence of APTES determined by TGA confirm the grafting of the APTES molecules on Pal surface.

SEM micrographs of untreated palygorskite clearly exhibit a fibrous

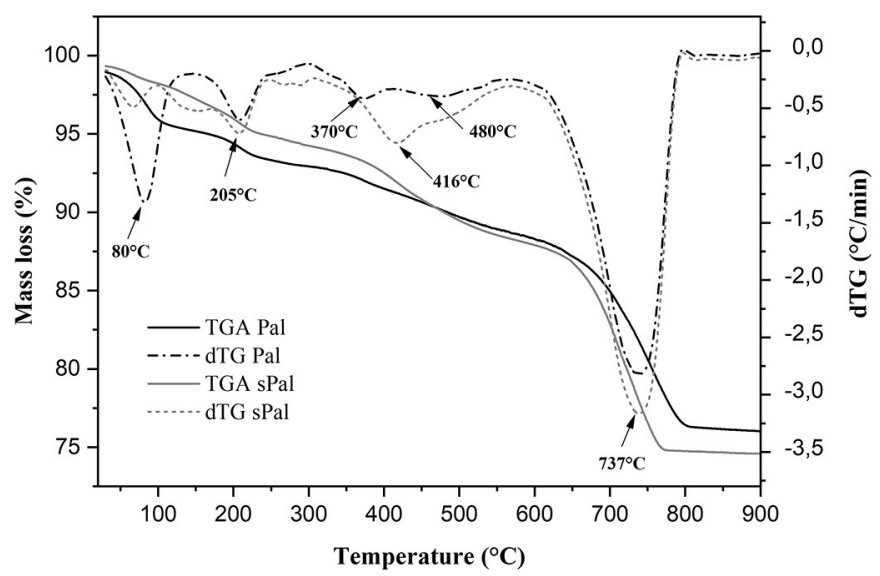

Fig. 2. TGA thermograms of Pal and sPal.

morphology (Fig. 3a-b), in which aggregates of entangled fibers are randomly oriented. This is due probably to strong physical and hydrogen bonding interactions between $\mathrm{OH}$ groups of Pal fibers. After treatment, the silanized palygorskite shows less aggregates compared to the unmodified one. The fibrous clay is less compact and the fibers are easily separated from each other. This phenomenon is due to a partial substitution of $\mathrm{OH}$ groups by organic APTES molecules inhibiting the hydrogen bonds formation between the Pal fibers. Indeed, the organic molecules, which are grafted on the surface of Pal fibers, can reduce the surface energy and the fibers-fibers interactions. This is consistent with the morphological data reported by Zhang et al. (Zhang et al., 2016)' indicating that clay aggregates can be broken down to single fibers after organic modification. 

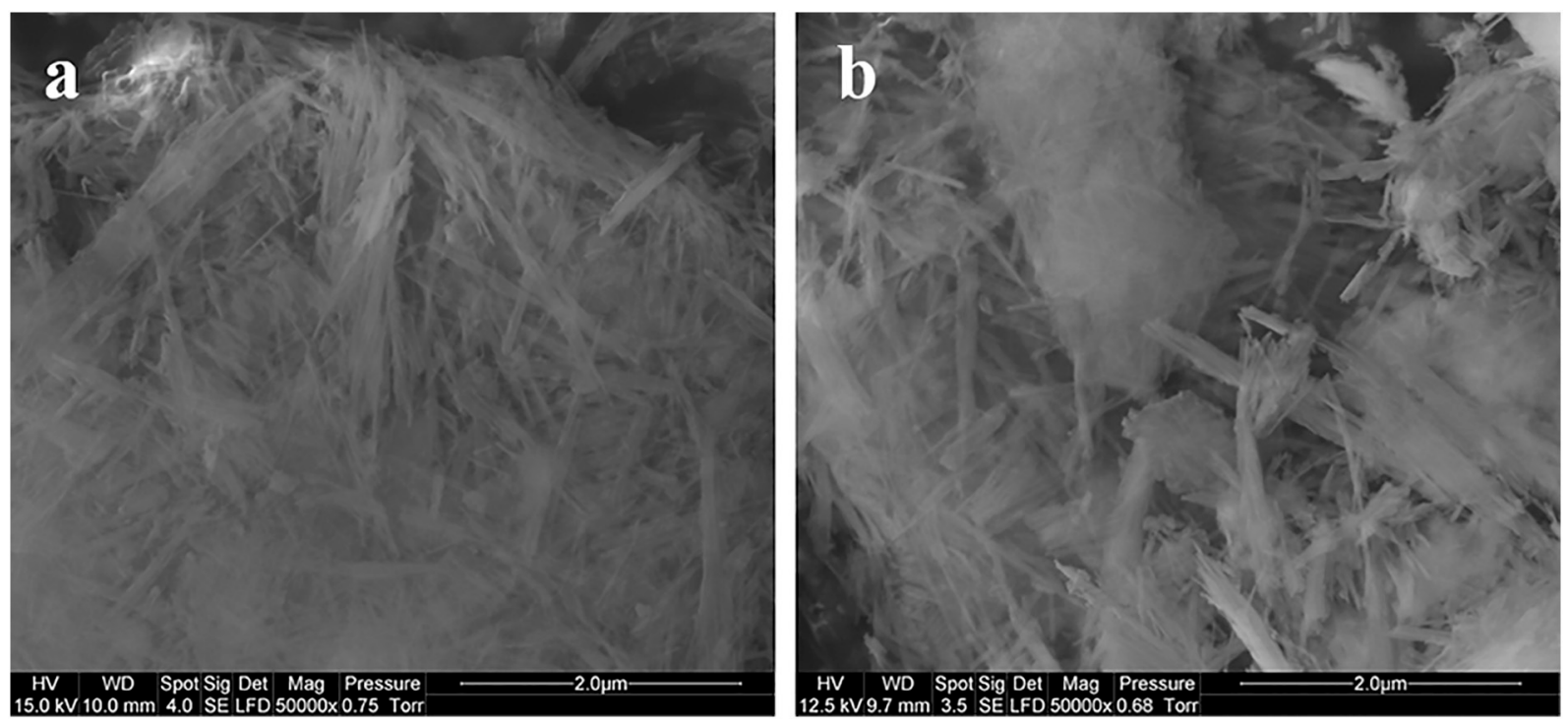

Fig. 3. SEM micrographs of Pal (a) and sPal (b) nanofillers.

3.2. Characterization of the thermal stability and the fire behaviour of the PA11-Pal nanocomposites

\subsubsection{Thermal stability}

TGA and DTG curves of neat PA11 and various PA11 nanocomposites in nitrogen and air atmospheres are shown in Figs. 4 and 5, respectively. The thermal decomposition of neat PA11 starts at $420{ }^{\circ} \mathrm{C}$ under nitrogen and $410{ }^{\circ} \mathrm{C}$ in the air. The thermal degradation mechanism occurs in a single step, with the maximum degradation rate observed at $460{ }^{\circ} \mathrm{C}$ whatever the atmosphere. The addition of MPP obviously affects the decomposition process of PA11, resulting in a change in the decomposition kinetics and the appearance of a second degradation step. The maximum degradation temperature of PA11-MPP was reduced compared to that of pure PA11, from $460{ }^{\circ} \mathrm{C}$ for PA11 to $385^{\circ} \mathrm{C}$ for PA11-MPP in inert atmosphere and from 460 to $380{ }^{\circ} \mathrm{C}$ in the air, respectively. The degradation step is due to the decomposition of MPP at lower temperatures. The second step occurs at the maximum decomposition rate of $434{ }^{\circ} \mathrm{C}$ corresponding to the degradation of the polymer chains.

However, the incorporation of $5 \mathrm{wt} \%$ of both Pal and sPal increases the degradation temperature of PA11 nanocomposites whatever the atmosphere. Indeed, the maximum decomposition temperature increases from $460{ }^{\circ} \mathrm{C}$ for the neat PA1 1 to almost $467{ }^{\circ} \mathrm{C}$ in the presence of both Pal and sPal.

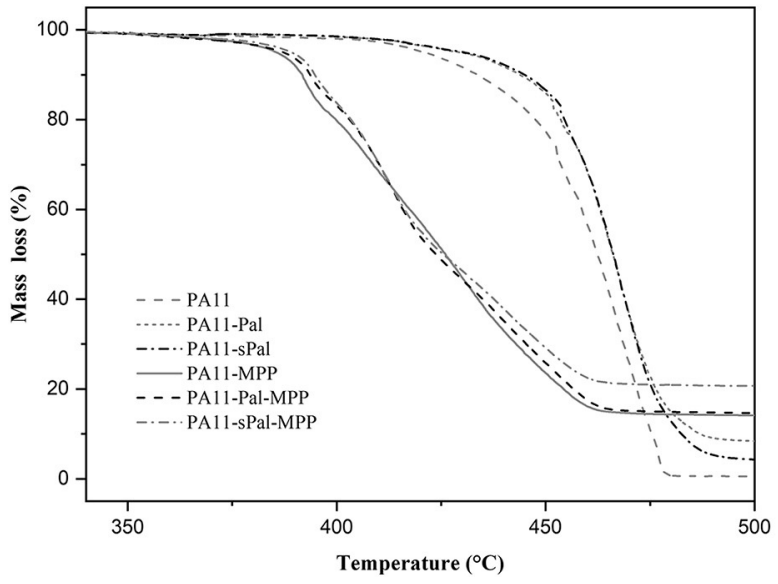

Furthermore, the decomposition of PA11-Pal-MPP and PA11-sPalMPP nanocomposites start at lower temperatures than that of pure PA11, due to the presence of MPP. Like the nanocomposite PA11-MPP, the decomposition of the ternary systems based on PA11-Pal-MPP and PA11-sPal-MPP proceeds in two steps. The various degradation steps observed in the samples containing MPP are due to the decomposition of MPP into melamine phosphate and melamine pyrophosphate which occurs above $380^{\circ} \mathrm{C}$. The degradation products are further decomposed into phosphoric acid and melamine (Jahromi et al., 2003; Wang et al., 2007; Rakotomalala et al., 2010). An additional degradation step at about $411{ }^{\circ} \mathrm{C}$ is also observed in the ternary system. This phenomenon is due probably to interactions and synergistic effect between Pal nanoparticles and MPP molecules. Similar behaviour is also observed under air atmosphere at almost the same temperature. The nature of these interactions is not well estabilished. However, according to the literature data (Braun et al., 2007, 2010), the appearance of an additional decomposition step is attributed to the additives interactions resulting in the formation of new phosphate structures. No effect of sPal on the thermal stability of PA11 nanocomposites is noticed. This could be due probably to either the low grafting ratio of APTES molecules on the Pal surface or the nanofiller content used, which are not enough to observe any noticeable effect of APTES on the thermal stability of PA11 nanocomposites.

Fig. 4. TGA and DTG curves of the nanocomposites under nitrogen.

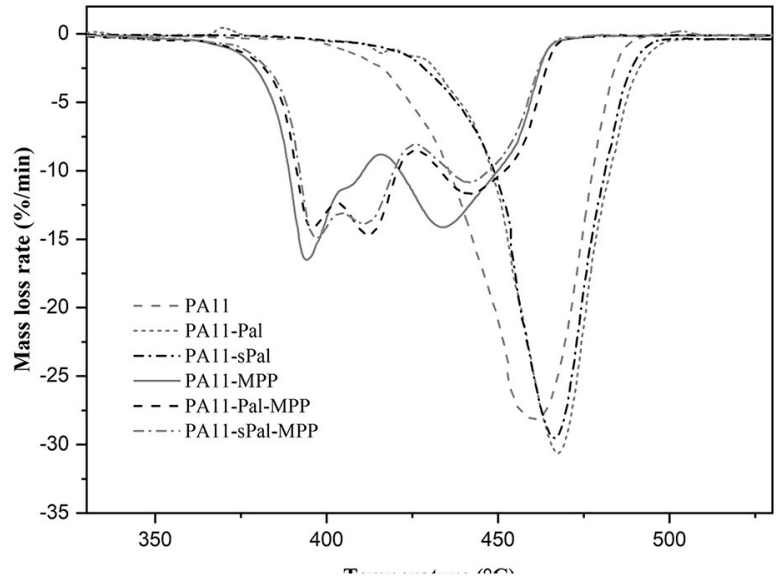



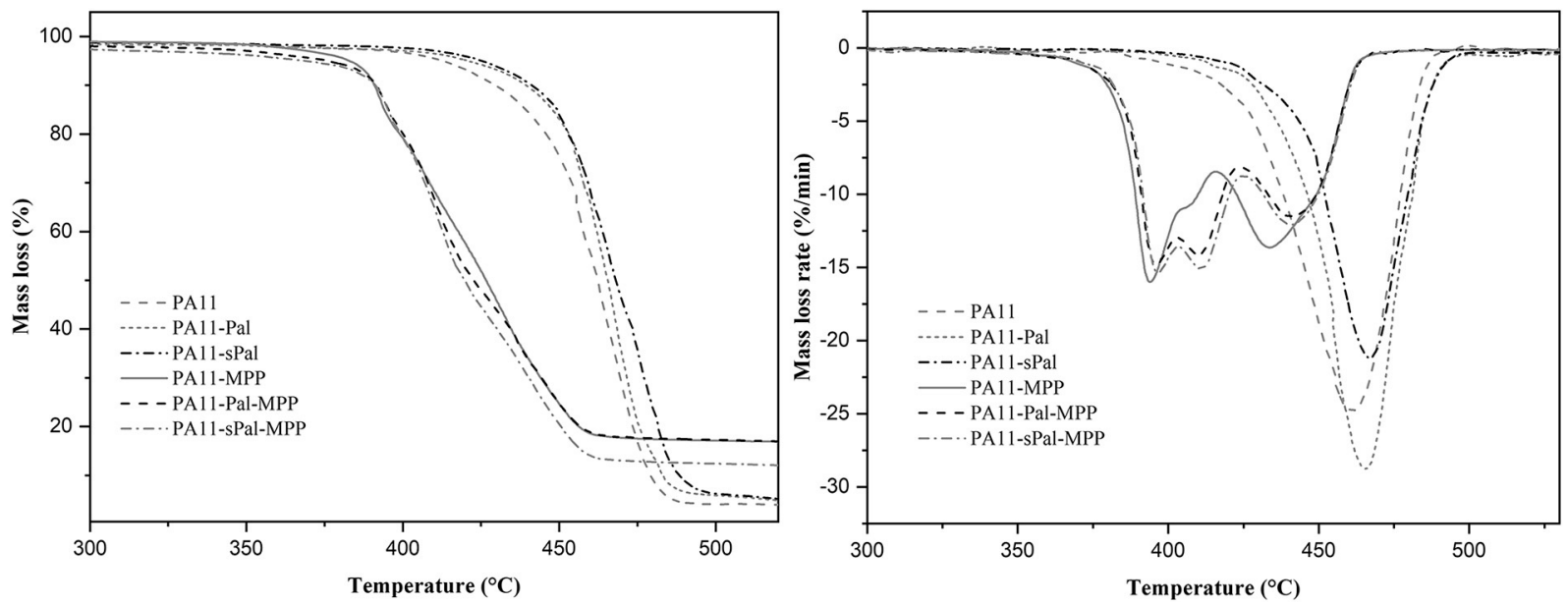

Fig. 5. TGA and DTG curves of the nanocomposites under air.

\subsubsection{Pyrolysis combustion flow calorimetry (PCFC)}

The plots of heat release rate (HRR) versus temperature of neat PA11 and its nanocomposites under anaerobic and aerobic conditions are presented in Fig. 6. The addition of Pal or sPal to PA11 causes a significant decrease in pHRR at a high temperature in both nitrogen and air atmosphere (Fig. 6 a and b), compared to the initial values of PA11. This decrease is however more pronounced in the presence of sPal, especially under air atmosphere. The addition of MPP results in the degradation of PA11 and the formation of two pHRR (Fig. 6 a and b) at a lower temperature. The first pHRR is due to the decomposition of MPP at $400{ }^{\circ} \mathrm{C}$, while the main peak of PA11 is significantly lowered by the flame-retardant activity. The combination of Pal or sPal with MPP entails a slight increase in pHRR and $\mathrm{T}_{\mathrm{pHRR}}$. The reduction of $\mathrm{pHRR}$ with increasing $\mathrm{T}_{\mathrm{pHRR}}$ by adding Pal and particularly sPal, indicates the good insulating effect of clays to the degradation of PA11. Furthermore, a charred structure could have been formed under nitrogen and air atmospheres.

\subsubsection{Cone calorimeter}

The cone calorimeter data of the samples are given in Tables 2 and 3 and the HRR curves obtained under heat flux of 35 and $50 \mathrm{~kW} / \mathrm{m}^{2}$ are shown in Fig. 7 a and b, respectively.

From the data given in Table 4 and illustrated in Fig. 7 a, (35 kW/ $\mathrm{m}^{2}$ irradiation), it is noticed that the neat PA11 burns rapidly after ignition and releases a very large amount of heat with a maximum value of $\mathrm{pHRR}=1054 \mathrm{~kW} / \mathrm{m}^{2}$. The PA11 nanocomposites burn less

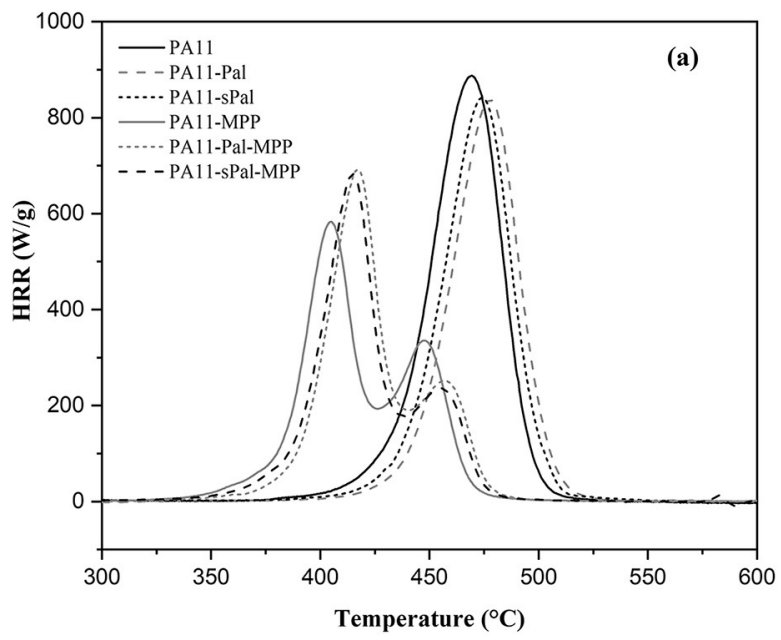

Table 2

Fire characteristics data from the cone calorimeter test recorded at $35 \mathrm{~kW} / \mathrm{m}^{2}$ for neat PA11 and its nanocomposites.

\begin{tabular}{lllllll}
\hline Samples & TTI (s) & $\begin{array}{l}\text { pHRR } \\
\left(\mathrm{kW} / \mathrm{m}^{2}\right)\end{array}$ & $\begin{array}{l}\text { Time } \\
\text { pHRR }(\mathrm{s})\end{array}$ & $\begin{array}{l}\text { THR } \\
(\mathrm{MJ} / \\
\left.\mathrm{m}^{2}\right)\end{array}$ & $\begin{array}{l}\text { MARHE } \\
\left(\mathrm{kW} / \mathrm{m}^{2}\right)\end{array}$ & Residue (\%) \\
\hline PA11 & 183 & 1054 & 327 & 140 & 349 & 0 \\
PA11-Pal & 185 & 798 & 415 & 136 & 282 & 5.37 \\
PA11-sPal & 184 & 716 & 350 & 137 & 289 & 4.28 \\
PA11-MPP & 98 & 676 & 358 & 128 & 298 & 6.56 \\
$\begin{array}{c}\text { PA11-Pal- } \\
\text { MPP }\end{array}$ & 97 & 598 & 277 & 129 & 338 & 11.2 \\
$\begin{array}{c}\text { PA11-sPal- } \\
\text { MPP }\end{array}$ & 93 & 580 & 287 & 130 & 303 & 10.6 \\
\hline
\end{tabular}

intensively, resulting in a reduction in pHRR value with levelling off of HRR curves. Compared with the neat PA11, the pHRR is significantly reduced by 24 and $32 \%$ after adding Pal and sPal, respectively. Furthermore, the time of flame out is higher than that of neat PA11, the good insulating effect of $\mathrm{Pal}$ and sPal could be responsible for the hindrance of PA11 combustion. The incorporation of MPP in PA11 matrix leads to a decrease in pHRR by almost 35\%. Indeed, the combination of $20 \mathrm{wt} \%$ of MPP and $5 \mathrm{wt} \%$ of Pal or sPal leads to a noticeable decrease in pHRR of PA11 nanocomposites, i.e., a reduction by $43 \%$ for PA11-Pal-MPP and 45\% for PA11-sPal-MPP in comparison with neat PA11. This reduction in pHRR could be the result of the

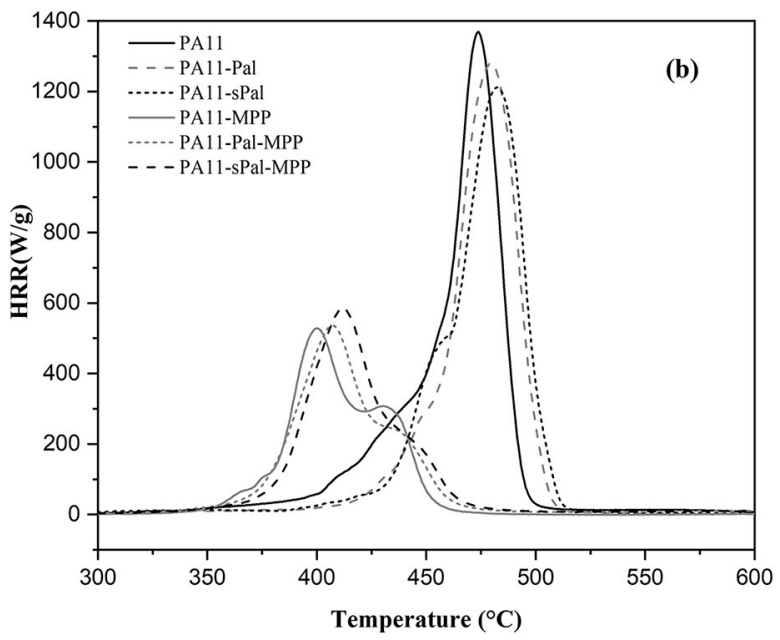

Fig. 6. PCFC curves under nitrogen (a) and air (b) of different compositions. 
Table 3

Fire characteristics data from the cone calorimeter test recorded at $50 \mathrm{~kW} / \mathrm{m}^{2}$ for neat PA11 and its nanocomposites.

\begin{tabular}{lllllll}
\hline Samples & TTI (s) & $\begin{array}{l}\text { pHRR } \\
\left(\mathrm{kW} / \mathrm{m}^{2}\right)\end{array}$ & $\begin{array}{l}\text { Time } \\
\text { pHRR }(\mathrm{s})\end{array}$ & $\begin{array}{l}\text { THR } \\
(\mathrm{MJ} / \\
\left.\mathrm{m}^{2}\right)\end{array}$ & $\begin{array}{l}\text { MAHRE } \\
\left(\mathrm{kW} / \mathrm{m}^{2}\right)\end{array}$ & Residue (\%) \\
\hline PA11 & 83 & 1420 & 235 & 148 & 550 & 0 \\
PA11-Pal & 82 & 1020 & 245 & 143 & 490 & 3.91 \\
PA11-sPal & 83 & 1011 & 255 & 141 & 470 & 3.65 \\
PA11-MPP & 53 & 865 & 260 & 135 & 448 & 6.85 \\
$\begin{array}{c}\text { PA11-Pal- } \\
\begin{array}{c}\text { MPP } \\
\text { PA11-sPal- }\end{array}\end{array}$ & 48 & 740 & 195 & 134 & 464 & 9.88 \\
$\begin{array}{c}\text { MPP } \\
\text { 48 }\end{array}$ & 732 & 220 & 135 & 465 & 10.22 \\
\hline
\end{tabular}

formation of a charred structure acting as a barrier effect against the heat transfer from the flame to underlying polymer and the combustible gas from pyrolysis zone to the flame (Sonnier et al., 2011).

The addition of melamine polyphosphate (MPP) to PA11 matrix significantly decreases the ignition time (TTI) due to the reactivity effect of the MPP on the PA11 chain. It is worthy to note that the burning behaviour during cone calorimeter experiment is different between adding Pal (and sPal) or MPP. In the presence of Pal or sPal, the materials swell with the formation of a protective layer before the flame ignites. However, in the presence of MPP, the materials first melt by bubbling, and then a thin carbonaceous layer is formed at the top of the bubbling surface before ignition. This behaviour is due probably to the decomposition of MPP molecules releasing melamine and phosphoric acid, which results in breaking PA11 chains. The phosphoric acid catalyses the char layer formation in the condensed phase and the melamine acts as blowing agent, increasing the thickness of char layer, thus protecting the polymer against the heat (Coquelle et al., 2015).

By increasing the heat flux $\left(50 \mathrm{~kW} / \mathrm{m}^{2}\right)$, the time to ignition is reduced (Table 3) and the materials burn faster at a higher heat release rate. Similar combustion behaviour as $35 \mathrm{~kW} / \mathrm{m}^{2}$ irradiations is noticed. In fact, the pHRR values decrease for all compositions compared to neat PA11, suggesting that even at a high heat flux up to $50 \mathrm{~kW} / \mathrm{m}^{2}$ (Fig. $7 \mathrm{~b}$ ), the effect of palygorskite on the nanocomposites flame retardancy remains unchanged and the flammability of the nanocomposites is strongly dependent on the final char structure.

In conclusion, all samples show a decrease in pHRR, THR (total heat release) and MARHE (maximum average release of heat emission) in comparison with those of neat PA11. These results clearly indicate that the addition of Pal, sPal, MPP and a combination of these additives to PA11 matrix improve the flame retardancy performance of PA11 nanocomposites.

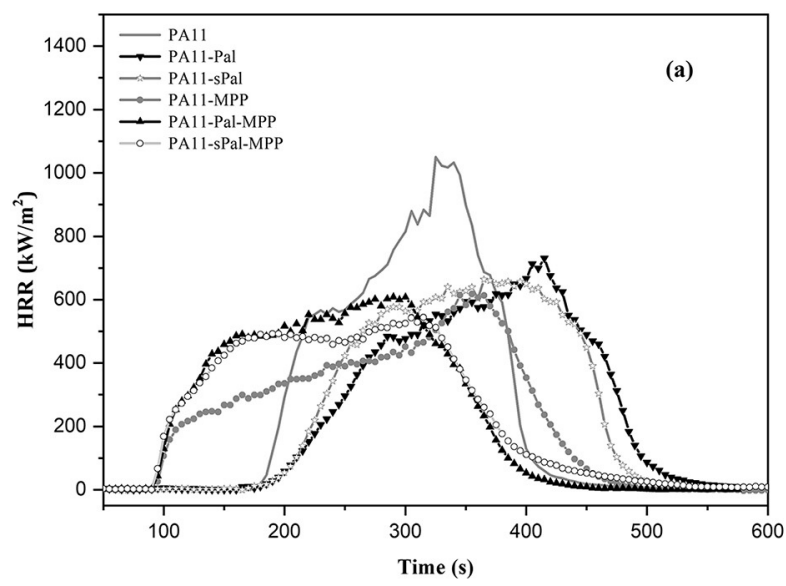

Table 4

Elemental composition of the char residue at $35 \mathrm{~kW} / \mathrm{m}^{2}$ and $50 \mathrm{~kW} / \mathrm{m}^{2}$ irradiations.

\begin{tabular}{|c|c|c|c|c|c|c|}
\hline \multicolumn{4}{|c|}{ Heat flux of $35 \mathrm{~kW} / \mathrm{m}^{2}$} & \multicolumn{3}{|c|}{ Heat flux of $50 \mathrm{~kW} / \mathrm{m}^{2}$} \\
\hline Samples & PA11-MPP & $\begin{array}{l}\text { PA11- } \\
\text { Pal-MPP }\end{array}$ & $\begin{array}{l}\text { PA11- } \\
\text { sPal-MPP }\end{array}$ & PA11-MPP & $\begin{array}{l}\text { PA11- } \\
\text { Pal-MPP }\end{array}$ & $\begin{array}{l}\text { PA11- } \\
\text { sPal-MPP }\end{array}$ \\
\hline $\mathrm{C}$ & 0,9 & 1,5 & 1,15 & 0,5 & 1,4 & 1 \\
\hline $\mathrm{O}$ & 28 & 44 & 48 & 32 & 41 & 45 \\
\hline $\mathrm{P}$ & 53 & 79 & 80 & 58 & 80 & 81 \\
\hline $\mathrm{N}$ & 8 & 2,3 & 2,1 & 7 & 2,5 & 2,5 \\
\hline
\end{tabular}

\subsubsection{Characterization of the char residue}

In order to verify whether the formation of carbonaceous char affects the combustion of the flame retardant of PA11 nanocomposites, some images on the char morphology have been taken using a digital camera after the cone calorimeter test. In this regard, Fig. 8 shows the digital pictures of residues of neat PA11 and its nanocomposites under heat flux of $35 \mathrm{~kW} / \mathrm{m}^{2}$. It is clearly observed that the neat PA11 is totally burnt. However, on adding the flame retardant, a char layer is formed on the surface of all nanocomposite samples. The char layer formed after burning of PA11-Pal and PA11-sPal samples is homogeneous. But, the char layer is thin and brittle indicating a limited protective action against heat and combustible gas transfer due to the low amount of residue. Conversely, the surface of PA11-MPP residue is continuous and compact. For PA11-Pal-MPP and PA11-sPal-MPP systems, a compact char is formed without cracks after combustion, due to the synergistic effect of Pal and MPP in PA11.

Accordingly, the combination of sPal or Pal with MPP promotes the formation of compact and thermally stable barrier which limits the heat and gas transfer, enhancing thereby the fire behaviour of PA11 systems.

The FT-IR spectra of cone calorimeter residue at $35 \mathrm{~kW} / \mathrm{m}^{2}$ irradiation are shown in Fig. 9. The absorption bands at 1010, 1050 and $1051 \mathrm{~cm}^{-1}$ are assigned to $\mathrm{Si}-\mathrm{O}$ in the burned palygorskite. The absorption bands at 941,945 and $948 \mathrm{~cm}^{-1}$ appearing in the samples containing MPP are attributed to $\mathrm{P}=\mathrm{O}$ groups (Sut et al., 2019). In addition, there are small peaks observed at 1240 and $1433 \mathrm{~cm}^{-1}$ assigned to the stretching vibration of $\mathrm{P}-\mathrm{N}$ and $\mathrm{P}-\mathrm{C}$ bonds, respectively. These bands are due to the formation of phosphate, phosphoric esters and polyphosphoric acid in the condensed phase in the samples containing MPP (Braun et al., 2010), as a result of the chemical interactions between Pal and MPP. The peaks at 1637 and $1620 \mathrm{~cm}^{-1}$ are due to $\mathrm{C}=\mathrm{C}$ bonds contained in the aromatic compounds (Su et al., 2011; Tai et al., 2012). Moreover, the same peaks are observed in the residue at $50 \mathrm{~kW} / \mathrm{m}^{2}$ irradiation (not shown). This suggests that the intensity of heat flux does not affect the chemical composition of the residue after burning.

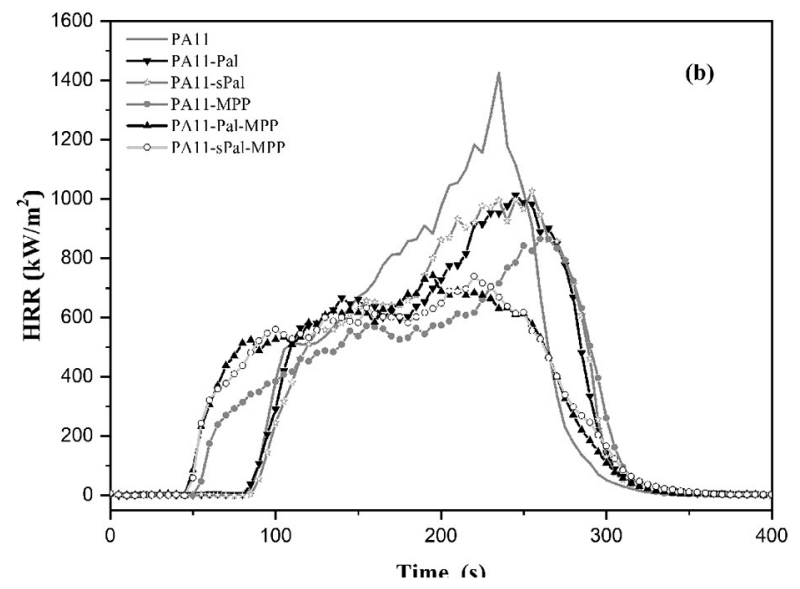

Fig. 7. HRR curves of PA11 and its nanocomposites under a heat flux $35 \mathrm{~kW} / \mathrm{m}^{2}$ (a) and $50 \mathrm{~kW} / \mathrm{m}^{2}$ (b). 

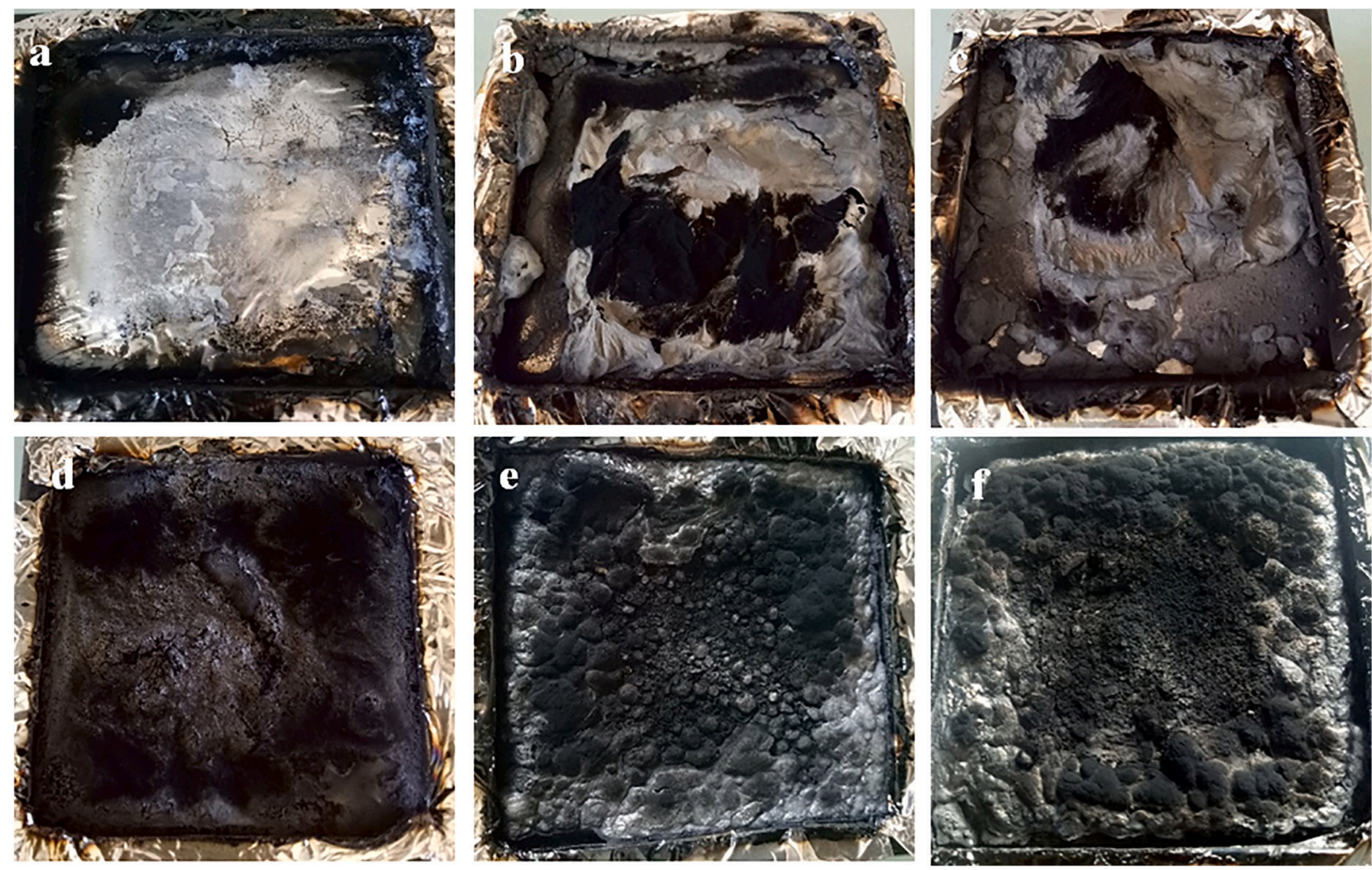

Fig. 8. Digital pictures of char residues after cone calorimeter test at $35 \mathrm{~kW} / \mathrm{m} 2$; a) PA11, b) PA11-Pal, c) PA11-sPal, d) PA11-MPP, e) PA11-Pal-MPP, f) PA11-sPalMPP.

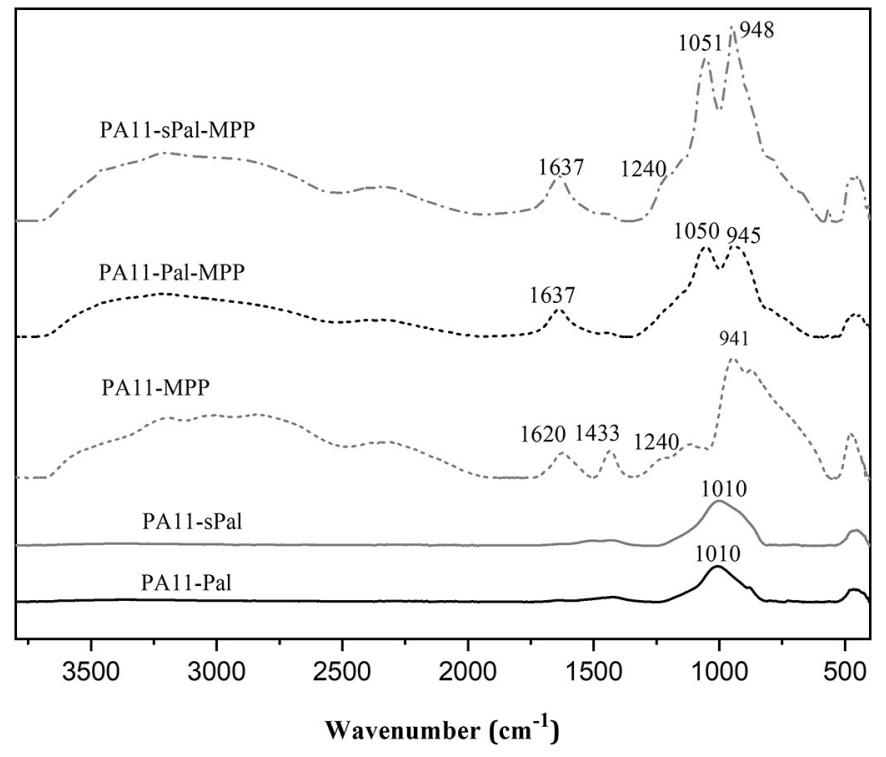

Fig. 9. FT-IR spectra of the residue at $35 \mathrm{~kW} / \mathrm{m}^{2}$ irradiation.

Table 4 gives the elemental analysis data of the residue after the cone calorimeter test. The results show that $\mathrm{P}$ and $\mathrm{N}$ elements still remain in the final chars after burning being that $\mathrm{P}$ is the predominant element in the condensed phase. In addition, the carbon concentration in ternary composites is higher than that of PA11-MPP system, whatever the heat flux, which points out the excellent carbonization ability of Pal and MPP combination, that is able to trap more phosphorus

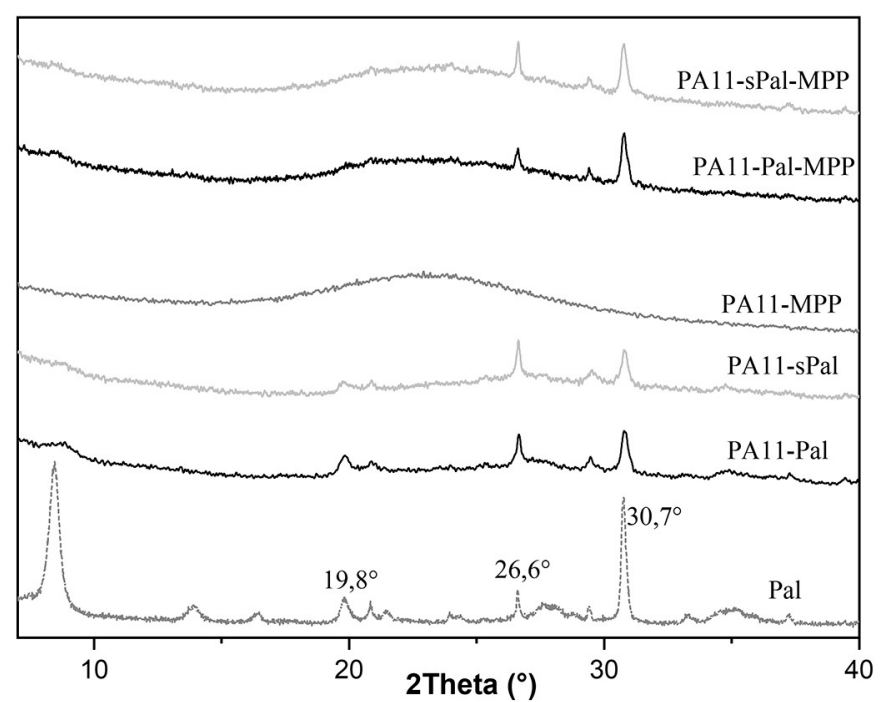

Fig. 10. WAXS patterns of char residue for the nanocomposites at heat flux of $35 \mathrm{~kW} / \mathrm{m}^{2}$.

element in the condensed phase.

WAXD patterns of the residue at $35 \mathrm{~kW} / \mathrm{m}^{2}$ irradiation (Fig. 10) and $50 \mathrm{~kW} / \mathrm{m}^{2}$ (not shown) exhibit similar crystalline peaks. After burning, the most characteristic peak of Pal disappeared, due probably to the dehydroxylation of Palygorskite structure at high temperature, indicating the folding of the Pal structure (Wang and Chen, 2013). However, some of crystalline peaks present in the reinforcing clay before burning still remain in the residue, especially those around $19.8^{\circ}$ 
and $26.6^{\circ}$, which belong to the quartz mineral (Zuo et al., 2016) and the pic at around $30,7^{\circ}$ corresponds to the dolomite (Boudriche et al., 2011), present in the raw clay and did not totally eliminate after purification with acidic attack.

Despite the high crystalline structure of MPP (not shown), it becomes completely amorphous after burning due to the degradation of melamine at a high temperature (Jahromi et al., 2003), which explains the amorphous structure of PA11-MPP char. No peaks corresponding to new crystalline phases are observed in PA11-Pal-MPP and PA11-sPalMPP residues, which exhibit similar peaks as those observed in PA11Pal, PA11-sPal and PA11-MPP residue.

\section{Conclusion}

Algerian palygorskite was successfully modified by APTES as supported by FT-IR and TGA data. The fire retardancy properties of neat PA11 in the presence of combined Pal or sPal with MPP were also investigated. The cone calorimeter and PCFC data showed that the addition of Pal and sPal led to improved fire retardancy of PA11 nanocomposites compared to neat PA11, in particular the pHRR, which was further reduced with the addition of MPP. Indeed, the cone calorimeter data showed that the combination of either Pal or sPal with MPP resulted in a better improvement in fire behaviour, due to the synergistic effect between Pal and MPP, in particular with the sinalized Pal. TGA data indicated an increase in thermal stability by adding Pal and sPal either under inert or air atmosphere, whereas, MPP caused a significant thermal degradation of PA11 matrix. The increase in phosphorus content in the condensed phase of PA11 nanocomposites containing both palygorskite and MPP compared to PA11- MPP, showed that MPP palygorskite the combination enhanced the flame retardancy action by trapping the phosphorus element in the condensed phase. This could be attributed to the synergistic effect of Pal and MPP in PA11, being however more pronounced with sPal.

\section{Author contributions}

The manuscript is a part of the Ph.D thesis of BB who has conducted the experimental work as well as the interpretation of the results and writing.

$\mathrm{AB}$ has conceptualized and supervised the work.

MK has reviewed, edited and validated the manuscript.

$J$-ML-C received BB in his laboratory for scientific internships several times, especially for the study of the fire behaviour of PA11 nanocomposites. He also reviewed and validated the manuscript.

\section{Declaration of Competing Interest}

The authors declare that they have no known competing financial interests or personal relationships that could have appeared to influence the work reported in this paper.

\section{Acknowledgements}

The authors are thankful to Benjamin Gallard, Sylvain Buonomo and Loic Dumazert of C2MA/IMT-Mines Ales (France) for their help to carry out the experimental work.

\section{References}

Belaroui, L.S., Ouali, A., Bengueddach, A., Lopez Galindo, A., Peña, A., 2018. Adsorption of linuron by an Algerian palygorskite modified with magnetic iron. Appl. Clay Sci. 164, 26-33. https://doi.org/10.1016/j.clay.2018.03.021.

Boudriche, L., Calvet, R., Hamdi, B., Balard, H., 2011. Effect of acid treatment on surface properties evolution of attapulgite clay: an application of inverse gas chromatography. Colloids Surfaces A Physicochem. Eng. Asp. 392, 45-54. https://doi.org/10. 1016/j.colsurfa.2011.09.031.

Braun, U., Schartel, B., Fichera, M.A., Jager, C., 2007. Flame retardancy mechanisms of aluminium phosphinate in combination with melamine polyphosphate and zinc borate in glass-fibre reinforced polyamide 6, 6. Polym. Degrad. Stab. 92, 1528-1545. https://doi.org/10.1016/j.polymdegradstab. 2007.05.007.

Braun, U., Bahr, H., Schartel, B., 2010. Fire retardancy effect of aluminium phosphinate and melamine polyphosphate in glass fibre reinforced polyamide 6. e-Polymers 10 , 443. https://doi.org/10.1515/epoly.2010.10.1.443.

Chen, F., Lou, D., Yang, J., Zhong, M., 2011. Mechanical and thermal properties of attapulgite clay reinforced polymethylmethacrylate nanocomposites. Polym. Adv. Technol. 22, 1912-1918. https://doi.org/10.1002/pat.1691.

Chen, L., Liu, K., Jin, T.X., Chen, F., Fu, Q., 2012. Rod like attapulgite/poly (ethylene terephthalate) nanocomposites with chemical bonding between the polymer chain and the filler. Express Polym Lett 6, 629-638. https://doi.org/10.3144/ expresspolymlett.2012.67.

Coquelle, M., Duquesne, S., Casetta, M., Sun, J., Gu, X., Zhang, S., Bourbigot, S., 2015. Flame retardancy of PA6 using a guanidine sulfamate/melamine polyphosphate mixture. Polymers (Basel) 7, 316-332. https://doi.org/10.3390/polym7020316.

Frini-Srasra, N., Srasra, E., 2010. Acid treatment of south Tunisian palygorskite : removal of Cd (II) from aqueous and phosphoric acid solutions. Desalination 250, 26-34. https://doi.org/10.1016/j.desal.2009.01.043.

Hao, A., Wong, I., Wu, H., Lisco, B., Ong, B., Sallean, A., Butler, S., Londa, M., Koo, J.H. 2015. Mechanical, thermal, and flame-retardant performance of polyamide 11 halloysite nanotube nanocomposites. J. Mater. Sci. 50, 157-167. https://doi.org/10. 1007/s10853-014-8575-7.

Hou, W., Fu, Y., Zeng, C., Liu, N., Yin, C., 2018. Enhancement of fl ame retardancy and mechanical properties of polyamide 6 by incorporating melamine cyanurate combined with attapulgite. J. Appl. Polym. Sci. 1-10. https://doi.org/10.1002/app. 47298.

Jahromi, S., Gabrie, W., Braam, A., 2003. Effect of melamine polyphosphate on thermal degradation of polyamides : a combined X-ray diffraction and solid-state NMR study Polymer (Guildf) 44, 25-37.

Jin, X., Chen, C., Sun, J., Zhang, X., GU, X., Zhang, S., 2016. The synergism between melamine and expandable graphite on improving the flame retardancy of polyamide 11. High Perform. Polym. 1-10. https://doi.org/10.1177/0954008316628965.

Jin, X., Sun, J., Zhang, J.S., Gu, X., Bourbigot, S., Li, H., Tang, W., Zhang, S., 2017. The preparation of a novel intumescent flame retardant based on supramolecular interactions and its application in polyamide 11. Appl. Mater. Interfaces 1-29.

Kaci, M., Dehouche, N., Focke, W.W., van der Merwe, E.M., 2019. A degradation study of polyamide 11/vermiculite nanocomposites under accelerated UV test. Polym. Eng. Sci. 60, 1-9. https://doi.org/10.1002/pen.25115.

Koo, J.H., Lao, S., Yong, W., Wu, C., Tower, C., Wissler, G.E., Pilato, L.A., Luo, Z., Scientist, C., 2008. Material characterization of intumescent flame retardant polyamide 11 nanocomposites. Am. Inst. Aeronaut. Astronaut. 1-14.

Lao, S.C., Wu, C., Moon, T.J., Koo, J.H., Morgan, A., Pilato, L., Wissler, G., 2009. Flame retardant polyamide11 and 12 nanocomposites: thermal and flammability properties. J. Compos. Mater. 43, 1803-1818. https://doi.org/10.1177/0021998309338413.

Lao, S., Kan, M., Lam, C., Chen, D., Koo, J., Moon, T., Londa, M., Takatsuka, T., Kuramoto, E., Wissler, G., Pilato, L., Luo, Z., 2010a. Polyamide 11-carbon nanotubes nanocomposites: processing, morphological, and property characterization. Solid Free. Fabr. Symp. 451-467.

Lao, S.C., Yong, W., Nguyen, K., Moon, T.J., Koo, J.H., Pilato, L., Wissler, G., 2010b. Flame-retardant polyamide 11 and 12 nanocomposites: processing, morphology, and mechanical properties. J. Compos. Mater. https://doi.org/10.1177/ 0021998310369580.

Lao, S.C., Koo, J.H., Moon, T.J., Londa, M., Ibeh, C.C., Wissler, G.E., Pilato, L.A., 2011. Flame-retardant polyamide 11 nanocomposites: further thermal and flammability studies. J. Fire Sci. 29, 479-498. https://doi.org/10.1177/0734904111404658.

Levchik, S.V., Costa, L., Camino, G., 1992. Effect of the fire-retardant, ammonium polyphosphate, on the thermal decomposition of aliphatic polyamides: part 11-polyamide 6. Polym. Degrad. Stab. 36, 229-237.

Macheca, A.D., Focke, W.W., Kaci, M., Panampilly, B., Androsch, R., 2017. Flame retarding polyamide 11 with exfoliated vermiculite nanoflakes. Polym. Eng. Sci. https://doi.org/10.1002/pen.24775.

Moreira, M.A., Ciuf, K.J., Rives, V., Vicente, M.A., Trujillano, R., Gil, A., Korili, S.A., De Faria, E.H., 2017. Effect of chemical modi fi cation of palygorskite and sepiolite by 3aminopropyltriethoxisilane on adsorption of cationic and anionic dyes. J. Appl. Polym. Sci. 135, 394-404. https://doi.org/10.1016/j.clay.2016.10.022.

Negrell, C., Frénéhard, O., Sonnier, R., Dumazert, L., Briffaud, T., Flat, J.-J., 2016. Selfextinguishing bio-based polyamides. Polym. Degrad. Stab. 134, 10-18. https://doi. org/10.1016/j.polymdegradstab. 2016.09.022.

Pan, B., Yue, Q., Ren, J., Wang, H., Jian, L., Zhang, J., Yang, S., 2006. A study on attapulgite reinforced PA6 composites. Polym. Test. 25, 384-391. https://doi.org/10. 1016/j.polymertesting.2005.11.012.

Qi, Z., Ye, H., Xu, J., Peng, J., Chen, J., Guo, B., 2013. Synthesis and characterizations of attapulgite reinforced branched poly(butylene succinate) nanocomposites. Colloids Surfaces A Physicochem. Eng. Asp. 436, 26-33. https://doi.org/10.1016/j.colsurfa. 2013.06.019.

Rakotomalala, M., Wagner, S., Dö, M., 2010. Recent developments in halogen free flame retardants for epoxy resins for electrical and electronic applications. Materials (Basel) 3, 4300-4327. https://doi.org/10.3390/ma3084300.

Rybiński, P., Janowska, G., Jóźwiak, Małgorzata, Jóźwiak, Marek, 2013. Thermal stability and flammability of styrene-butadiene rubber (SBR) composites. J. Therm. Anal. Calorim. 113, 43-52. https://doi.org/10.1007/s10973-012-2871-8.

Sahnoune, M., Taguet, A., Otazaghine, B., Kaci, M., Lopez-Cuesta, J.M., 2018. Fire retardancy effect of phosphorus-modified halloysite on polyamide- 11 nanocomposites. Polym. Eng. Sci. 1-9. https://doi.org/10.1002/pen.24961.

Sonnier, R., Ferry, L., Longuet, C., Laoutid, F., Friederich, B., 2011. Combining cone 
calorimeter and PCFC to determine the mode of action of fl ame - retardant additives. Polym. Adv. Technol. 22, 1091-1099. https://doi.org/10.1002/pat.1989.

Su, X.Q., Tao, J., Yi, Y.W., Cui, Y.H., Wang, Q., 2011. Synergistic flame retardant effect of attapulgite on Pp/App/Per compersites. In: 18th Int. Conf. Compos. Mater. 1-6.

Sut, A., Metzsch-Zilligen, E., Großhauser, M., Pfaendner, R., Schartel, B., 2019. Synergy between melamine cyanurate, melamine polyphosphate and aluminum diethylphosphinate in flame retarded thermoplastic polyurethane. Polym. Test. 74, 196-204. https://doi.org/10.1016/j.polymertesting.2019.01.001.

Tai, Q., Yuen, R.K.K., Yang, W., Qiao, Z., Song, L., Hu, Y., 2012. Composites : part a ironmontmorillonite and zinc borate as synergistic agents in flame-retardant glass fiber reinforced polyamide 6 composites in combination with melamine polyphosphate. Compos. Part A 43, 415-422. https://doi.org/10.1016/j.compositesa.2011.10.012.

Wang, J., Chen, D., 2013. Mechanical properties of natural rubber nanocomposites filled with thermally treated attapulgite. J. Nanomater. 1-11.

Wang, Z., Feng, Z., Liu, Y., Wang, Q., 2007. Flame retarding glass fibers reinforced polyamide 6 by melamine polyphosphate/polyurethane-encapsulated solid acid. J. Appl. Polym. Sci. 105, 3317-3322. https://doi.org/10.1002/app.

Wang, C., Wu, Q., Liu, F., An, J., Lu, R., Xie, H., Cheng, R., 2014. Synthesis and characterization of soy polyol-based polyurethane nanocomposites reinforced with silylated palygorskite. Appl. Clay Sci. 101, 246-252. https://doi.org/10.1016/j.clay. 2014.08.009.

Yang, F., Nelson, G.L., 2011. Combination effect of nanoparticles with flame retardants on the flammability of nanocomposites. Polym. Degrad. Stab. 96, 270-276. https://doi. org/10.1016/j.polymdegradstab. 2010.06.003.

Yuan, X., Li, C., Guan, G., Liu, X., Xiao, Y., Zhang, D., 2007. Synthesis and characterization of poly(ethylene terephthalate)/attapulgite nanocomposites. J. Appl. Polym. Sci. 103, 449-456. https://doi.org/10.1002/app.

Zhang, Q., Yu, M., Fu, Q., 2004. Crystal Morphology and Crystallization Kinetics of Polyamide-11/Clay Nanocomposites 1949. pp. 1941-1949. https://doi.org/10.1002/ pi.1581.

Zhang, Y., Wang, B., Hu, G., 2012. Isothermal crystallization kinetics and melting behavior of polyamide 11/silica nanocomposites prepared by in situ melt polymerization. J. Appl. Polym. Sci. 123, 273-279. https://doi.org/10.1002/app.

Zhang, Y., Zhao, J., Chu, H., Zhou, X., Wei, Y., 2014. Effect of modified attapulgite addition on the performance of a PVDF ultra fi ltration membrane. Desalination 344, 71-78. https://doi.org/10.1016/j.desal.2014.03.007.

Zhang, Y., Yu, C., Hu, P., Tong, W., Lv, F., Chu, P.K., Wang, H., 2016. Mechanical and thermal properties of palygorskite poly (butylene succinate) nanocomposite. Appl. Clay Sci. 119, 96-102. https://doi.org/10.1016/j.clay.2015.07.022.

Zhu, L., Guo, J., Liu, P., Zhao, S., 2016. Novel strategy for palygorskite/poly (acrylic acid) nanocomposite hydrogels from bi-functionalized palygorskite nanorods as easily separable adsorbent for cationic basic dye. Appl. Clay Sci. 121-122, 29-35. https://doi org/10.1016/j.clay.2015.12.020.

Zuo, R., Du, G., Yang, W., Liao, L., Li, Z., 2016. Mineralogical and chemical characteristics of a powder and purified quartz from Yunnan Province. Open Geosci. 8, 606-611. https://doi.org/10.1515/geo-2016-0055. 